\title{
FORMAÇÃO E ESTABILIZAÇÃO DE AGREGADOS DO SOLO INFLUENCIADOS POR CICLOS DE UMEDECIMENTO E SECAGEM APÓS ADIÇÃO DE COMPOSTOS ORGÂNICOS COM DIFERENTES CARACTE RÍSTICAS HIDROFÓBICAS ${ }^{(1)}$
}

\author{
Renato Saldanha Bastos ${ }^{(2)}$, Eduardo de Sá Mendonça(3), \\ Víctor Hugo Alvarez V.(3), Marcelo Metri Corrêa ${ }^{(4)}$ \& \\ Liovando Marciano da Costa(3)
}

\begin{abstract}
RESUMO
A adição de compostos orgâni cos e os ciclos de umedeci mento e secagem do solo têm influência marcante na agregação do solo. Este trabalho objetivou estudar o efei to do tempo de incubação e a influência dos ciclos de umedeci mento e secagem sobre a ação de compostos orgânicos com características hidrofóbicas e hidrofílicas na agregação de um Latossolo Vermelho-Amarelo distrófico. Os tratamentos dos experimentos de períodos de incubação e de ciclos de umedecimento e secagem seguiram esquema fatorial incompleto $2 \times 3[(4-1)+(4-$ 1)]. Os fatores foram: TFSA de dois horizontes ( $A$ e $B$ ), três períodos de incubação (40, 80 e 160 dias), três compostos orgânicos (amido, ácido esteárico e ácido húmico), em combinações de ausência e presença de amido e ácido húmico (Am e AH), (4-1) e de ácido esteárico e ácido húmico (E e AH), (4-1). Todas as unidades experimentais foram mantidas em incubadora de DBO a $25^{\circ} \mathrm{C}$ e em ambiente natural, quando foram submetidas a 5, 11 e 22 ciclos de umedecimento e secagem, de acordo com os períodos de incubação. Após incubação, as amostras de agregados foram analisadas quanto ao diâmetro médio ponderado, diâmetro médio geométrico, índice de estabilidade de agregados e grau de repelência à água. Os resultados indicaram que a aplicação, mesmo em longos períodos de incubação, de compostos or gâni cos com caráter hidrofóbico acentuado mostrou efeito marcante na melhoria da estabilidade em água do agregados, sendo sua melhor resposta na dose de 5,5 $\mathrm{g} \mathrm{kg}^{-1}$. Para melhorar a agregação, a aplicação
\end{abstract}

\footnotetext{
(1) Trabal ho extraído da Tese de Mestrado do primeiro autor, em Solos e Nutrição de Plantas pela U niversidade F ederal de Viçosa UFV. Recebido para publicação em setembro de 2003 e aprovado em novembro de 2004.

(2) Mestre em Solos e Nutrição de Plantas pela Universidade Federal de Viçosa- UFV. Departamento de Solos, CEP $36570-000$ Viçosa (MG).

(3) Professor do Departamento de Solos, UFV. E-mails: esm@ufv.br; vhav@ufv.br; liovandomc@yhaoo.com.br

(4) Doutorando em Solos e Nutrição de Plantas, Departamento de Solos, UFV. E-mail: marcelometri@yahoo.com
} 


\begin{abstract}
de compostos orgânicos hidrofílicos deve ser contínua e com períodos de incubação de no máximo $\mathbf{4 0}$ dias. Os ciclos de umedecimento e secagem reduzi ram os efeitos dos compostos orgânicos adicionados sobre a agregação.
\end{abstract}

Termos de indexação: agregação, gênese, ácidos orgânicos, solos tropicais.

\author{
SUMMARY: SOIL AGGREGATE FORMATION AND STABILIZATION \\ AS INFLUENCED BY WETTING DRYING CYCLES AND \\ ORGANIC COMPOUNDS WITH DIFFERENT HYDROPHOBIC \\ CHARACTERISTICS
}

\begin{abstract}
The input of organic compounds in agricultureand the soil wetting and drying cycles havea strong influence on soil aggregation. This study investigated the effect of incubation periods and theinfluence of wetting and drying cycles with theaddition of organic compounds of distinct hydrophobic and hydrophilic characteristics on theaggregation of a Red-Yellow Latosol. The experiments were arranged in an incompletefactorial design $2 \times 3[(4-1)+(4-$ 1)]. The factors were: air-dried soil of $A$ and $B$ horizons, three incubation periods (40, 80 and 160 days), three organic compounds (amid, estearic acid, and humic acid), the combination of presence and absence of amid/ acid humic (Am $+\mathrm{AH}),(4-1)$ and estearic acid/ humic acid $(E+A H),(4+1)$. All experimental units werekept in a BOD incubator at $25^{\circ} \mathrm{C}$ and in natural environment, wherethey underwent 5,11 and 22 wetting and drying cycles. After the incubation period, the aggregate samples were analyzed for aggregate stability index, mean weight diameter, mean geometric diameter, and water repel lenced egree Results indicated that even in the long term the application of organic compounds with strong hydrophobic character contributes considerably to theimprovement of water aggregate stability. The $5.5 \mathrm{~g} \mathrm{~kg}^{-1}$ dose achieved best results. To improve the aggregation, the application of hydrophilic organic compounds should be continuous in intervals of up to 40 days. Soil wetting and drying cycles reduced the effect of organic compounds on aggregation.
\end{abstract}

Index terms: aggregation, genesis, organic acids, tropical soils.

\section{NTRODUÇÃO}

Agregados são componentes da estrutura do solo e, portanto, de suma importância na manutenção da por osi dade e aeração do solo, no crescimento das plantas e da população microbiana, na infiltração de água e no control e dos processos erosivos (Oades, 1984; Dexter, 1988).

Para a formação do agregado, é necessário que os colóides do solo se encontrem floculados e que todos os componentes do agregado sejam posteriormente estabilizados por algum agente cimentante (Hillel et al., 1980). Os agregados podem ser classificados quanto ao tamanho, de acordo com a teoria da hierarquização de agregados (Tisdall \& Oades, 1982), em cinco grupos (<2 $\mu \mathrm{m}$; de 2 a $20 \mu \mathrm{m}$; de 20 a $250 \mu \mathrm{m}$, de $250 \mu \mathrm{m}$ a $2 \mathrm{~mm}$; e $>2 \mathrm{~mm}$ ). Os menores que $250 \mu \mathrm{m}$ são denominados microagregados e os maiores, macroagregados, sendo cada agregado formado pela união dos agregados da classe que vem logo abaixo, seguindo, assim, uma ordem hierárquica. Conforme seus tamanhos, cada classe será unida por diferentes agentes cimentantes.

Graças à forteação cimentante dos óxidos de ferro e de alumínio nos macroagregados, a teoria da hierarquização não se aplica completamente aos Latossolos (Oxissol os) (Oades \& Waters (1991). O mesmo pode ocorrer em solos cauliníticos, com presença de óxidos de ferro e de alumínio de baixa cristalinidade (Six et al., 2000).

Diversos são os fatores que interferem na agregação do solo. Dentreesses, os principais são: tipo e teor deargila, metais polivalentes, carbonato de cálcio, óxidos e hidróxidos de ferro, alumínio e manganês, exudatos orgâni cos de plantas, substâncias orgânicas provenientes da ação de microrganismos e outros compostos orgânicos. A matéria orgânica, os óxidos e os hidróxidos são agentes cimentantes, tanto das partículas primárias quanto das partículas secundárias do solo. Podem-se incluir, também, os ciclos de umedecimento e secagem como importante fator ambiental que interfere na agregação do solo (Carvalho, 1991 \& Maltoni, 1994). 
A matéria orgânica, funcionando como agente cimentante do solo, tem si do foco de vários trabalhos (Baver, 1968; Tisdall \& Oades, 1982; Chaney \& Swift, 1984; Silva \& Mielniczuk, 1998; Castro Filho et al., 1998). O aumento da estabilidade dos agregados está intimamente relacionado com a capacidade da matéria orgânica de se aderir às partículas minerais do solo, formando as ligações argilo-metal-húmicas (E dwrds \& Bremner, 1967).

Os agentes orgâni cos envol vidos na estabi lização dos agregados do solo podem ser divididos em três grupos, quanto a sua resistência à ação mi crobiana: os transicionais, os temporários e os persistentes. Os primeiros são os polissacarídeos que são rapidamente decompostos pelos microrganismos (Angers \& Mehuys, 1989) e, normalmente, estão associados à estabilidade dos macroagregados do solo. Os agentes temporários são as hifas de fungos e raízes, que permanecem no solo por semanas, meses ou até anos, estão associados aos macroagregados. As raízes podem apresentar ação mecânica, promovendo pressão nos constituintes do solo, e química por meio das secreções. Os agentes persistentes são os materiais orgâni cos humi ficados, principalmente associados aos óxidos de Fe e Al de baixa cristalinidade, constituindo a parte mais importante na formação de mi croagregados do solo (Tisdall \& Oades, 1982).

O objetivo deste trabalho foi estudar o efeito do tempo de incubação e dos ciclos de umedecimento e secagem sobre a ação de compostos orgânicos hidrofóbicos e hidrofílicos na agregação do solo.

\section{MATERIAL E MÉTODOS}

Foram utilizadas amostras dos horizontes $A$ $(0-20 \mathrm{~cm})$ eB $(40-60 \mathrm{~cm})$ de um L atossoloVermel hoAmarelo distrófico (LVAd) da região de Viçosa, Minas Gerais (Bastos et al., 2005).

O período experimental estendeu-se entre os meses de novembro de 2001 e maio de 2002, caracterizado como verão quente e úmido, com temperaturas variando de 20 a $37^{\circ} \mathrm{C}$.

\section{Experimento de períodos de incubação}

Os tratamentos do experimento de períodos de incubação seguiriam o esquema fatorial incompleto $2 \times 3[(4-1)+(4-1)]$, cujos fatores foram: dois horizontes (A e B), três tempos de incubação (40, 80 e 160 dias), três compostos orgânicos (amido, ácido esteárico e ácido húmico) (Bastos et al., 2005), em combinações de ausência e presença deamido eácido húmico (Am e AH), (4-1) e de áci do esteárico eácido húmico (E e $A H)$, (4-1). O delineamento experimental utilizado foi inteiramentecasualizado com três repetições. Foram utilizadas as doses 0,0 ou de $9,9 \mathrm{~g} \mathrm{~kg}^{-1}$ de cada composto orgânico e nos tratamentos $\mathrm{Am}+\mathrm{AH}$ e $\mathrm{E}+\mathrm{AH}$ a dose de cada composto foi de $4,45 \mathrm{~g} \mathrm{~kg}^{-1}$, resultando em uma dose total de $9,9 \mathrm{~g} \mathrm{~kg}^{-1}$.

Para a realização do trabalho, $100 \mathrm{~g}$ de TFSA foram colocados em potes plásticos com tampa de vol ume aproximado de $250 \mathrm{~mL}$. Todos os compostos foram homogeneizados e adicionados em pó ao solo seco. Em seguida, adicionou-se a cada pote água deionizada atéatingir $80 \%$ da capacidade decampo, 29,36 dag kg-1, no horizonte A, e 30,48 dag kg-1, no horizonte $B$. Todos os potes foram tampados e mantidos em incubadora de DBO a $25{ }^{\circ} \mathrm{C}$ pelos períodos de tempo de 40, 80 ou 160 dias. Semanalmente, todos os potes foram retirados da incubadora eabertos, para quesereduzisse a pressão de $\mathrm{CO}_{2}$ interna, e, concomitantemente, fez-se um rearranjo dos potes, assim reduzindo a desuniformidade das condições no interior da incubadora.

Após o término do período de incubação, as unidades amostrais foram retiradas da incubadora e os recipientes deixados abertos durante dez dias, a fim de que as amostras atingissem o equilíbrio com a umidade atmosférica. Em seguida, as amostras foram anal isadas quanto às características físicas e morfológicas dos agregados do solo.

\section{Experimento de ciclos de umedecimento e secagem}

O experimento de ciclos de umedecimento e secagem baseou-se no mesmo esquema fatorial, fatores, delineamento experimental e doses usadas no experimento de períodos de incubação. Nesse caso, o sol o foi submeti do a cicl os de umedecimento e secagem semanais, totalizando 5, 11 e 22 ciclos para os períodos de incubação de 40, 80 ou 160 dias. Para isto, $100 \mathrm{~g}$ de TFSA foram colocados em potes plásticos com tampa de volume aproximado de 250 $\mathrm{mL}$. Todos os compostos foram adicionados em pó ao solo seco e homogeneizados. Em seguida, adicionou-se em cada pote água deionizada até atingir $80 \%$ da capacidade de campo, 29,36 dag kg-1, no horizonte A, e 30,48 dag kg$^{-1}$, no horizonte B. Os potes foram mantidos abertos em local sombreado e arejado. A quantidade de água perdida em uma semana era determinada por gravimetria e reposta atéatingir novamente $80 \%$ da capaci dade de campo. Ao final dos períodos de incubação, o reumedecimento era suspenso e, após 10 dias, o solo foi analisado quanto às mesmas características do experimento de períodos de incubação.

\section{Variáveis morfológicas avaliadas via seca}

Para avaliar a estabilidade deagregados via seca, foi utilizado o método indireto de peneiramento a seco. Este procedimento foi realizado para a separação inicial dos agregados formados. Foram utilizadas duas peneiras (4,00 e 2,00 mm) e um vibrador Produtest, agitando-sea amostra, durante 
5 min, na graduação no 5 do aparelho. A seguir, parte de cada fração foi seca em estufa a $105^{\circ} \mathrm{C}$ para correção de seu peso. Assim, calcularam-se o diâmetro médio ponderado (DMP) e o diâmetro médio geométrico (DMG), segundo equações propostas por Kemper \& Chepil (1965).

\section{Variáveis morfológicas avaliadas via úmida}

Para avaliar a estabilidade de agregados via úmida, foram usados $20 \mathrm{~g}$ de solo, os quais foram pré-umedecidos, conforme o princípio de umedecimento lento descrito por Kemper \& Chepil (1965). Depois desse pré-umedecimento, a amostra foi mantida em repouso à temperatura ambiente por 30 min. Logo em seguida, essa amostra foi posta no aparel ho de oscilação vertical sobre um conjunto de peneiras de 2,00; 1,00; 0,50; 0,25 e 0,105 mm de diâmetro, conforme descrito por Yoder (1936). Transcorridos $15 \mathrm{~min}$, as porções retidas em cada peneira foram transferidas para cápsulas de alumínio com o auxílio de jatos de água, e secas em estufa a $105^{\circ} \mathrm{C}$ por um período de $24 \mathrm{~h}$ para posterior pesagem. A partir dos valores dessas massas, foram calculados o DMP , oDMG e o índice de estabilidade de agregados (IEA), conforme descrito por Kemper $\&$ Chepil (1965).

O grau de repelência à água dos agregados foi avaliado pel o método do tempo de penetração da gota d'água (Letey, 1969). Aproximadamente, $20 \mathrm{~g}$ desolo foram colocados em um prato e a superfície foi alisada com a mão. Nove gotas d'água, cada uma com 0,033 $\pm 0,00202 \mathrm{~mL}$ foram colocadas ao acaso sobre o solo seco ao ar e os tempos de infiltração cronometrados. O tempo médio de infiltração das nove gotas foi utilizado para classificar os agregados quanto a seu grau de repelência, como proposto por King (1981).

\section{Análises estatísticas}

Os experimentos de períodos de incubação e de cicl os de umedeci mento e secagem foram anal isados por contrastes. Nestes, foram estudados o efeito do tempo de incubação dentro dos dois horizontes do solo LVAd e os efeitos dos tratamento dentro dos períodos de incubação e dentro dos horizontes.

Foram montados os contrastes de avaliação dos efeitos no fatorial incompleto $2 \times 3[(4-1)+(4-1)]$ (Quadro 1). Os graus de liberdade para tratamento foram decompostos em seis contrastes ortogonais, visando isolar o efeito dos materiais orgânicos. Optou-se para trabalhar com contrastes médios, dividindo-se o resultado de cada contraste por $(\Sigma|\mathrm{ci}| /$ 2 ), em que ci são os respectivos coeficientes ortogonais. A significância dos contrastes foi testada pelo teste $F(P<0,10, P<0,05$ e $P<0,01)$. Os contrastes foram com o auxilio do programa SAEG versão 5.0 (UFV, 1993).

\section{Quadro 1. Contrastes de avaliação dos efeitos dos tratamentos dos experimentos nos tempos de incubação e de ciclos de umedecimento e secagem}

\begin{tabular}{lccc}
\hline \multirow{2}{*}{ Composto orgânico } & \multicolumn{3}{c}{ Contraste } \\
\cline { 2 - 4 } & C 1 & C2 & C 3 \\
\hline Testemunha(1) & -3 & 0 & 0 \\
Am & + & - & - \\
$A H^{(1)}$ & + & - & + \\
$A m+A H$ & + & +2 & 0 \\
& & Contraste \\
\cline { 2 - 4 } & C4 & C5 & C6 \\
Testemunha(1) & -3 & 0 & 0 \\
A ${ }^{(1)}$ & + & - & - \\
E $+\mathrm{AH}$ & + & - & + \\
& + & +2 & 0
\end{tabular}

$\overline{\mathrm{Am}}=$ amido; $\mathrm{AH}=$ ácido húmico; $\mathrm{E}=$ ácido esteárico; $\mathrm{Am+ \textrm {AH }}=$ amido e ácido húmico; $\mathrm{E}+\mathrm{AH}$ = ácido esteárico e ácido húmico; $\mathrm{C} 1=\mathrm{T}$ vs $\mathrm{Am}+\mathrm{AH}+(\mathrm{Am}+\mathrm{AH}) ; \mathrm{C} 2=\mathrm{Am}+\mathrm{AH}$ vs $(\mathrm{Am}+\mathrm{AH})$; $\mathrm{C} 3=\mathrm{Am}$ vs $\mathrm{AH} ; \mathrm{C} 4=\mathrm{T}$ vs $\mathrm{E}+\mathrm{AH}+(\mathrm{E}+\mathrm{AH}) ; \mathrm{C} 5=\mathrm{E}+\mathrm{AH}$ vs $(\mathrm{E}+\mathrm{AH}) ; \mathrm{C} 6=\mathrm{E}$ vs $\mathrm{AH}$.

(1) Tratamento repetido somente uma vez por repetição [(4-1) + (4-1)].

\section{RESULTADOS E DISCUSSÃO}

\section{Efeito do período de incubação}

Os contrastes T vs Am + AH +(Am + AH) (C1) eT vs $\mathrm{E}+\mathrm{AH}+(\mathrm{E}+\mathrm{AH})(\mathrm{C} 4)$, que comparam a testemunha versus os outros tratamentos, foram positivos, indicando que todos os compostos orgânicos melhoraram a agregação do solo (Quadros 2, 3 e 4). Ressalta-se que, geralmente, as variáveis analisadas foram significativas no horizonte A nos períodos de 80 e 160 dias de incubação e em todos os tempos de incubação (40, 80 e 160 dias) na amostra do horizonte B. I sso mostra um efeito mais rápido dos compostos orgânicos na agregação do horizonte B. É provável que tal fato se deva à agregação no horizonte $A$ inicialmente maior e muito influenciada pelos materiais orgânicos nativos, que não estão presentes no horizonte B. Piccolo\& M bagwu (1999) mostraram que, num solo onde a matéria orgânica inicial foi removida, a ação agregante dos compostos orgânicos é mais pronunciada.

Nohorizonte B, comparando a testemunha versus o amido, o ácido húmico e a mistura dos dois (C1), percebeu-setendência de queda nos valores deDMP, DMG e IEA com o passar do tempo (Quadro 3). J á no contraste 4 (C4), que compara a testemunha versus o áci do esteárico, o ácido húmico e a mistura dos dois, observou-se tendência de diminuição da agregação no horizonte $A$, fato que se inverte no horizonte $B$, indicando que os compostos orgânicos com caráter hidrofóbico acarretam aumento na agregação nos períodos de incubação mais longos. 
Quadro 2. Diâmetros médios ponderado e geométrico de agregados de amostras dos horizontes A e B de um LVAd incubadas em temperatura controlada de $25^{\circ} \mathrm{C}$ e separadas por via seca

\begin{tabular}{|c|c|c|c|c|c|c|c|}
\hline \multirow{2}{*}{ Horizonte } & \multirow{2}{*}{$\begin{array}{c}\text { Período } \\
\text { de } \\
\text { incubação }\end{array}$} & \multicolumn{6}{|c|}{ Composto orgânico } \\
\hline & & $\mathbf{T}$ & Am & $\mathbf{E}$ & $\mathbf{A H}$ & $\mathbf{A m}+\mathbf{A H}$ & $\mathbf{E}+\mathbf{A H}$ \\
\hline & dia & \multicolumn{6}{|c|}{ Diâmetro médio ponderado, $\mathrm{mm}$} \\
\hline \multirow[t]{3}{*}{ A } & 40 & 4,35 & 4,05 & 4,04 & 6,75 & 6,05 & 5,96 \\
\hline & 80 & 3,62 & 2,86 & 4,19 & 7,31 & 6,81 & 6,87 \\
\hline & 160 & 3,79 & 2,76 & 3,94 & 7,09 & 6,52 & 5,65 \\
\hline \multirow[t]{4}{*}{$\mathrm{B}$} & 40 & 3,15 & 5,02 & 2,19 & 8,61 & 7,58 & 6,90 \\
\hline & 80 & 4,71 & 3,56 & 2,24 & 11,25 & 8,59 & 7,89 \\
\hline & 160 & 4,33 & 1,64 & 1,58 & 11,99 & 7,96 & 8,14 \\
\hline & \multicolumn{7}{|c|}{ Diâmetro médio geométrico, mm } \\
\hline \multirow[t]{3}{*}{ A } & 40 & 2,05 & 1,63 & 1,63 & 2,59 & 2,33 & 2,24 \\
\hline & 80 & 1,56 & 1,38 & 1,68 & 2,81 & 2,61 & 2,61 \\
\hline & 160 & 1,62 & 1,35 & 1,62 & 2,69 & 2,50 & 2,16 \\
\hline \multirow[t]{3}{*}{ B } & 40 & 1,43 & 1,90 & 1,23 & 3,36 & 2,83 & 2,59 \\
\hline & 80 & 1,82 & 1,52 & 1,24 & 5,12 & 3,34 & 2,99 \\
\hline & 160 & 1,78 & 1,15 & 1,12 & 5,40 & 3,01 & 3,13 \\
\hline
\end{tabular}

$\mathrm{T}$ = testemunha; $\mathrm{Am}=$ amido; $\mathrm{E}$ = ácido esteárico; $\mathrm{AH}$ =ácido húmico.

Quadro 3. Diâmetros médios ponderado e geométrico e índice de estabilidade de agregados de amostras dos horizontes A e B de um LVAd incubadas em temperatura controlada de $25^{\circ} \mathrm{C}$ e separadas por via úmida

\begin{tabular}{|c|c|c|c|c|c|c|c|}
\hline \multirow{2}{*}{ Horizonte } & \multirow{2}{*}{$\begin{array}{c}\text { Período } \\
\text { de } \\
\text { incubação }\end{array}$} & \multicolumn{6}{|c|}{ Composto orgânico } \\
\hline & & $\mathbf{T}$ & Am & $\mathbf{E}$ & $\mathbf{A H}$ & $\mathbf{A m}+\mathbf{A H}$ & $\mathbf{E}+\mathbf{A H}$ \\
\hline & dia & \multicolumn{6}{|c|}{ Diâmetro médio ponderado, mm } \\
\hline \multirow[t]{3}{*}{ A } & 40 & 0,89 & 0,85 & 0,90 & 0,87 & 1,00 & 0,91 \\
\hline & 80 & 0,81 & 0,89 & 0,96 & 0,99 & 1,06 & 1,06 \\
\hline & 160 & 0,84 & 0,91 & 0,97 & 0,94 & 1,02 & 1,06 \\
\hline \multirow[t]{4}{*}{ B } & 40 & 0,55 & 0,65 & 0,57 & 0,74 & 0,73 & 0,69 \\
\hline & 80 & 0,55 & 0,69 & 0,68 & 0,73 & 0,85 & 0,75 \\
\hline & 160 & 0,59 & 0,66 & 0,74 & 0,78 & 0,75 & 0,81 \\
\hline & \multicolumn{7}{|c|}{ Diâmetro médio geométrico, mm } \\
\hline \multirow[t]{3}{*}{ A } & 40 & 0,66 & 0,65 & 0,68 & 0,65 & 0,73 & 0,68 \\
\hline & 80 & 0,61 & 0,67 & 0,71 & 0,72 & 0,77 & 0,77 \\
\hline & 160 & 0,64 & 0,69 & 0,72 & 0,68 & 0,75 & 0,77 \\
\hline \multirow[t]{4}{*}{ B } & 40 & 0,43 & 0,50 & 0,47 & 0,58 & 0,49 & 0,54 \\
\hline & 80 & 0,45 & 0,53 & 0,54 & 0,55 & 0,63 & 0,56 \\
\hline & 160 & 0,49 & 0,51 & 0,58 & 0,59 & 0,56 & 0,60 \\
\hline & \multicolumn{7}{|c|}{ Índice de estabilidade de agregados,\% } \\
\hline \multirow[t]{3}{*}{ A } & 40 & 93,98 & 95,44 & 95,15 & 93,21 & 96,56 & 94,55 \\
\hline & 80 & 92,00 & 96,42 & 95,18 & 96,89 & 97,84 & 97,17 \\
\hline & 160 & 94,74 & 95,63 & 96,04 & 94,51 & 97,81 & 98,49 \\
\hline \multirow[t]{3}{*}{$B$} & 40 & 81,00 & 86,55 & 86,05 & 93,68 & 67,88 & 92,24 \\
\hline & 80 & 85,53 & 88,48 & 90,56 & 91,58 & 95,21 & 90,23 \\
\hline & 160 & 88,93 & 88,88 & 93,39 & 92,48 & 90,31 & 93,42 \\
\hline
\end{tabular}

T = testemunha; $\mathrm{Am}=$ amido; $\mathrm{E}$ =ácido esteárico; $\mathrm{AH}$ =ácido húmico. 
Por meio do contraste 2 (C2), observa-se que há maior ação da mistura do que propriamente dos compostos isolados, quando analisados por via úmida. Essa ação agregante reduz-se com o passar do tempo de incubação. Pelo contraste 5 (C5), observa-se que, no horizonte $B$, ao contrário do que ocorre no horizonte A, nas variáveis analisadas por via úmida, que melhores valores de agregação se dão nos maiores tempos de incubação.
Esses resultados indicam que períodos de incubação longos de solo com materiais orgânicos com caráter hidrofílico levam a uma diminuição na estabilidade dos agregados. Este comportamento deve estar relacionado com o consumo desses compostos orgânicos pela microbiota do solo, pois esses são metabol izados primeiramente (Stevenson \& Cole, 1999). Parte da recal citrância do composto hidrofóbico está relacionada com sua repelência à

Quadro 4. Contrastes médios para as variáveis analisadas no experimento de períodos de incubação

\begin{tabular}{|c|c|c|c|c|c|}
\hline Contraste & DMPS & DMGS & DMPU & DMGU & IEAU \\
\hline & \multicolumn{4}{|c|}{$\mathrm{mm} \longrightarrow$} & $\%$ \\
\hline$\overline{\mathrm{C}} 1 \mathrm{~d} / \mathrm{T} \mathrm{H}_{40} \mathrm{~d} / \mathrm{A}$ & 1,27 & 0,13 & 0,02 & 0,02 & 1,09 \\
\hline$\underline{\mathrm{C}} 1 \mathrm{~d} / \mathrm{T}_{80} \mathrm{~d} / \mathrm{A}$ & $2,04 *$ & $0,71^{*}$ & $0,17^{* *}$ & $0,11^{* *}$ & $5,05^{\circ}$ \\
\hline$\underline{-} \underline{1} \mathrm{~d} / \mathrm{T}_{160} \mathrm{~d} / \mathrm{A}$ & $1,67 *$ & $0,56^{\circ}$ & $0,12 * *$ & $0,06 *$ & 1,24 \\
\hline$\underline{-} 2 \mathrm{~d} / \mathrm{T}_{40} \mathrm{~d} / \mathrm{A}$ & 0,65 & 0,22 & $0,14^{* *}$ & $0,08 *$ & 2,24 \\
\hline $\bar{C} 2 \mathrm{~d} / \mathrm{T}_{80} \mathrm{~d} / \mathrm{A}$ & $1,73^{*}$ & 0,51 & $0,12 * *$ & $0,08 *$ & 1,19 \\
\hline$\underline{-} 22 d / T_{160} d / A$ & $1,60^{\circ}$ & 0,48 & $0,10 *$ & $0,07 *$ & 2,74 \\
\hline$\underline{-} 3 \mathrm{~d} / \mathrm{T}_{40} \mathrm{~d} / \mathrm{A}$ & $2,70 * *$ & $0,96^{*}$ & 0,02 & 0,00 & $-2,23$ \\
\hline$\underline{-} 3 \mathrm{~d} / \mathrm{T}_{80} \mathrm{~d} / \mathrm{A}$ & $4,45^{* *}$ & $1,43^{* *}$ & $0,10 *$ & 0,05 & 0,47 \\
\hline$\underline{-} 3 \mathrm{~d} / \mathrm{T}_{160} \mathrm{~d} / \mathrm{A}$ & $4,33^{* *}$ & $1,34 * *$ & 0,03 & $-0,01$ & $-1,12$ \\
\hline$\overline{\mathrm{C}} 1 \mathrm{~d} / \mathrm{T}_{40} \mathrm{~d} / \mathrm{B}$ & $3,92 * *$ & $1,27 * *$ & $0,16 * *$ & $0,09 * *$ & 1,70 \\
\hline$\underline{-} 1 \mathrm{~d} / \mathrm{T}_{80} \mathrm{~d} / \mathrm{B}$ & $3,09 * *$ & $1,51 * *$ & $0,21^{* *}$ & $0,12 * *$ & $6,23 *$ \\
\hline$\underline{-} 1 \mathrm{~d} / \mathrm{T}_{160} \mathrm{~d} / \mathrm{B}$ & $2,87^{* *}$ & $1,41^{* *}$ & $0,14^{* *}$ & $0,06^{*}$ & 1,63 \\
\hline$\overline{\mathrm{C}} 2 \mathrm{~d} / \mathrm{T}_{40} \mathrm{~d} / \mathrm{B}$ & 0,77 & 0,20 & 0,04 & $0,05^{*}$ & $22,24 * *$ \\
\hline$\underline{-}-2 d / T_{80} d / B$ & 1,19 & 0,02 & $0,14^{* *}$ & $0,09 * *$ & $5,18^{\circ}$ \\
\hline$\underline{-} 22 d / T_{160} d / B$ & 1,15 & 0,27 & 0,03 & 0,01 & $-0,37$ \\
\hline$\overline{\mathrm{C}} 3 \mathrm{~d} / \mathrm{T} 40 \mathrm{~d} / \mathrm{B}$ & $3,59 * *$ & $1,46 * *$ & $0,09^{\circ}$ & $0,08 *$ & $7,13^{*}$ \\
\hline$\underline{-} 3 \mathrm{~d} / \mathrm{T}_{80} \mathrm{~d} / \mathrm{B}$ & 7,69** & $3,60 * *$ & 0,04 & 0,02 & 3,10 \\
\hline$\underline{-} 3 \mathrm{~d} / \mathrm{T}_{160} \mathrm{~d} / \mathrm{B}$ & $10,35^{* *}$ & $4,25^{* *}$ & $0,12^{*}$ & $0,08 *$ & 3,60 \\
\hline$\overline{\mathrm{C}} 4 \mathrm{~d} / \mathrm{T}_{40} \mathrm{~d} / \mathrm{A}$ & 1,23 & 0,10 & 0,00 & 0,01 & 0,32 \\
\hline$\underline{-}-4 d / T_{80} d / A$ & $2,20 * *$ & $0,81 *$ & $0,19 * *$ & $0,12 * *$ & 4,41 \\
\hline$\underline{-} 4 \mathrm{~d} / \mathrm{T}_{160} \mathrm{~d} / \mathrm{A}$ & $1,77^{*}$ & $0,54^{\circ}$ & $0,15^{* *}$ & $0,08^{* *}$ & 1,61 \\
\hline$\overline{\mathrm{C}} 5 \mathrm{~d} / \mathrm{T}_{40} \mathrm{~d} / \mathrm{A}$ & 0,57 & 0,13 & 0,03 & 0,02 & 0,37 \\
\hline $\bar{C} 5 \mathrm{~d} / \mathrm{T}_{80} \mathrm{~d} / \mathrm{A}$ & 1,12 & 0,37 & $0,09^{\circ}$ & $0,06 *$ & 1,14 \\
\hline $\bar{C} 5 \mathrm{~d} / \mathrm{T}_{160} \mathrm{~d} / \mathrm{A}$ & 0,14 & 0,00 & $0,11^{*}$ & $0,07 *$ & 3,22 \\
\hline$\underline{-} 6 \mathrm{~d} / \mathrm{T}_{40} \mathrm{~d} / \mathrm{A}$ & $2,71 * *$ & $0,96^{*}$ & $-0,03$ & $-0,03$ & $-1,94$ \\
\hline$\underline{-}-6 d / T_{80} d / A$ & $3,12 * *$ & $1,13^{* *}$ & 0,03 & 0,01 & 1,71 \\
\hline$\overline{\mathrm{C}} 6 \mathrm{~d} / \mathrm{T}_{160} \mathrm{~d} / \mathrm{A}$ & $3,15^{* *}$ & $1,07 * *$ & $-0,03$ & $-0,04$ & $-1,53$ \\
\hline$\underline{-} 4 \mathrm{~d} / \mathrm{T}_{40} \mathrm{~d} / \mathrm{B}$ & $2,75^{* *}$ & $0,96 * *$ & $0,12^{* *}$ & $0,10 * *$ & $9,66^{* *}$ \\
\hline$\overline{\mathrm{C}} 4 \mathrm{~d} / \mathrm{T}_{80} \mathrm{~d} / \mathrm{B}$ & $2,41 * *$ & $1,30 * *$ & $0,17^{* *}$ & $0,10 * *$ & $5,26^{\circ}$ \\
\hline$\underline{\mathrm{C}} 4 \mathrm{~d} / \mathrm{T}_{160} \mathrm{~d} / \mathrm{B}$ & $2,91^{* *}$ & $1,44 * *$ & $0,19 * *$ & $0,10 * *$ & 4,16 \\
\hline$\overline{\mathrm{C}} 5 \mathrm{~d} / \mathrm{T}_{40} \mathrm{~d} / \mathrm{B}$ & $1,50^{\circ}$ & 0,30 & 0,03 & 0,02 & 2,38 \\
\hline$\underline{-} 5 \mathrm{~d} / \mathrm{T}_{80} \mathrm{~d} / \mathrm{B}$ & 1,15 & $-0,19$ & 0,05 & 0,02 & $-0,84$ \\
\hline $\bar{C} 5 \mathrm{~d} / \mathrm{T}_{160} \mathrm{~d} / \mathrm{B}$ & 1,35 & $-0,13$ & $0,05^{\circ}$ & 0,02 & 0,49 \\
\hline$\overline{\mathrm{C}} 6 \mathrm{~d} / \mathrm{T}_{40} \mathrm{~d} / \mathrm{B}$ & $6,42 * *$ & $2,13^{* *}$ & $0,17^{* *}$ & $0,11^{* *}$ & $7,63^{*}$ \\
\hline$\underline{\mathrm{C}} 6 \mathrm{~d} / \mathrm{T}_{80} \mathrm{~d} / \mathrm{B}$ & $9,01^{* *}$ & $3,88^{* *}$ & 0,05 & 0,01 & 1,02 \\
\hline$\overline{\mathrm{C}} 6 \mathrm{~d} / \mathrm{T}_{160} \mathrm{~d} / \mathrm{B}$ & $10,41^{* *}$ & $4,28^{* *}$ & 0,04 & 0,01 & $-0,91$ \\
\hline Resíduo $(72)^{+}$ & $1,40^{++}$ & $0,23^{++}$ & $0,003^{++}$ & $0,002^{++}$ & $16,03^{++}$ \\
\hline C.V. (\%) & 21,32 & 20,97 & 7,11 & 7,25 & 4,34 \\
\hline
\end{tabular}

DMPS = diâmetro médio ponderado via seca; DMGS = diâmetro médio geométrico via seca; DMPU = diâmetro médio ponderado via úmida; DMGU = diâmetro médio geométrico via úmida; IEAU = índice de estabilidade de agregados via úmida.

$\overline{\mathrm{C}} 1=[-3 \mathrm{~T}+\mathrm{Am}+\mathrm{AH}+(\mathrm{Am}+\mathrm{AH})] / 3 ; \overline{\mathrm{C}} 2=[-\mathrm{Am}-\mathrm{AH}+2(\mathrm{Am}+\mathrm{AH})] / 2 ; \overline{\mathrm{C}} 3=(-\mathrm{Am}+\mathrm{AH}) / 1 ; \overline{\mathrm{C}} 4=[-3 \mathrm{~T}+\mathrm{E}+\mathrm{AH}+(\mathrm{E}+\mathrm{AH})] / 3 ;$ $\overline{\mathrm{C}} 5=[-\mathrm{E}-\mathrm{AH}+2(\mathrm{E}+\mathrm{AH})] / 2 ; \overline{\mathrm{C}} 6=(-\mathrm{E}+\mathrm{AH}) / 1 ; \mathrm{d} /$ - dentro de; $\mathrm{T}_{40}, \mathrm{~T}_{80}$ e $\mathrm{T}_{160}$ - período de incubação de 40, 80 e 160 dias, respectivamente; $\mathrm{A}$ - horizonte $\mathrm{A} ; \mathrm{B}$ - horizonte $\mathrm{B} ;{ }^{\circ},{ }^{*}$ e $\mathrm{e}^{* *}$ - contraste significativo a 10,5 e $1 \%$ pel o teste $\mathrm{F}$, respectivamente; +e + - grau de liberdade e quadrado médio. 
água, dificultando a ação dos mi crorganismos. Esse comportamento indica que, para que o material orgânico hidrofílico tenha importância na formação emanutenção de agregados, ele deve ser adicionado continuamente e em períodos curtos, enquanto compostos com caráter hidrofóbico dominante podem ser adicionados em períodos mais longos.

O ácido húmico, quando comparado aos outros compostos orgânicos, tevesempreefeitopredominante e, na maioria das vezes, significativo, como visto nos contrastes amido versus ácido húmico (C3) e ácido esteárico versus ácido húmico (C6) (Quadro 4). O ácido húmico é uma molécula que apresenta, de forma marcante, tanto caráter hidrofílico, quanto hidrofóbico (Hayes \& Malcolm, 2001). Assim, esse pode interagir com a fase coloidal do solo por meio de ligações hidrofílicas e, ainda, exibir alguma repelência à água na superfície dos agregados, por conta da porção hidrofóbica da molécula, tornandoos menos propensos à desestruturação. Nas análises via seca, oácido húmico apresentou maior agregação no tempo de 80 dias, para o horizonte $A$, e de 160 dias, para o horizonte B (Quadro 2). Isso, provavelmente, ocorre porque sua eficiência na estabilização do sol o nãoé muito afetada pelo tempo ou pela microbiota, já que esse é um agente de agregação permanente (Tisdall \& Oades, 1982).

Nas análises via úmida, o efeito doáci do húmico foi parecido com o da análise via seca (Quadro 4). Ressalta-se que, enquanto os dados da via seca têm valores sempre maiores em $B$, nas análises via úmida isso se inverte (Quadros 2 e 3). Esses resultados indicam que a formação de agregados estáveis em água éinfluenciada pelo conteúdo inicial de matéria orgânica do solo. Tal comportamento deve estar relacionado com a natureza da matéria orgânica, que apresenta alta densidade de cargas, e ao mesmotempo, caráter hidrofóbi co em suas frações.

As elevadas doses de amido eácido esteárico tiveram efeito negativo sobre a formação de agregados. Val ores el evados de amido e principal mente de ácido esteárico dificultaram a formação de agregados estáveis em água. Contudo, observa-se que, nos contrastes em que são comparados o amido, o ácido húmico e a combinação destes contra a testemunha no horizonte $\mathrm{B}$, geralmente ocorre dimi nui ção na formação eestabi lização dos agregados nos mai ores tempos de incubação. Como observado por Tisdall \& Oades (1982), Chaney \& Swift (1986a) e Piccolo \& Mbagwu (1999), os polissacarídeos, compostos hidrofílicos, têm efeito passagei ro na agregação, portanto com o tempo o efeito diminui. Já o ácido esteárico, composto com caráter hidrofóbico, teve, geral mente, efeito negativo na agregação, quando foi analisado por via seca, e efeito positivo quando analisado por via úmida (Quadro 4). Esse resultado mostra que o ácido esteárico não é muito eficiente na formação de agregados grandes, como é o ácido húmico, porém aumenta a resistência à entrada de água no agregado e, conseqüentemente, sua estabilidade.

O horizonte A teveresultados distintos para cada tratamento quanto ao grau de repelência à água (Quadro 5). O amido provocou pequena repelência aos 160 dias de incubação, o que nãoteve efeito sobre a agregação, já que ocorreram os maiores índices de estabilidade aos 40 dias de incubação na análise via seca e aos 80 dias na análise via úmida.

Os tratamentos com o ácido esteárico, sozinho ou em combinação com o ácido húmico, mostraram maior repelência à água aos 160 dias de incubação. Tal hidrofobicidade foi bem relacionada com o efeito do áci do esteárico na estabilidade dos agregados, já

Quadro 5. Tempo de penetração de gota e classes de repelência à água dos solos para o experimento de períodos de incubação

\begin{tabular}{|c|c|c|c|c|}
\hline \multirow{2}{*}{ Horizonte } & \multirow{2}{*}{ Composto orgânico } & \multicolumn{3}{|c|}{ Tempo de incubação (dias) } \\
\hline & & 40 & 80 & 160 \\
\hline & & & $\mathrm{S}$ & - \\
\hline \multirow[t]{6}{*}{$A$} & $\mathrm{~T}$ & $0(N R)^{(1)}$ & $0(N R)$ & $0(N R)$ \\
\hline & Am & 0 (NR) & 0 (N R) & 9 (RF a) \\
\hline & $\mathrm{E}$ & 1 (NR) & $6(\mathrm{RF} a)$ & 940 (RS) \\
\hline & $\mathrm{AH}$ & $0(N R)$ & $1(\mathrm{NR})$ & 9 (RF a) \\
\hline & $A m+A H$ & 11 (RFa) & 32 (RF a) & 340 (RF o) \\
\hline & $\mathrm{E}+\mathrm{AH}$ & $18(\mathrm{RFa})$ & 70 (RFo) & 900 (RS) \\
\hline \multirow[t]{6}{*}{$\mathrm{B}$} & $\mathrm{T}$ & $0(\mathrm{NR})$ & $0(N R)$ & $0(N R)$ \\
\hline & Am & $0(N R)$ & $0(N R)$ & $0(N R)$ \\
\hline & $\mathrm{E}$ & $0(N R)$ & $0(N R)$ & $0(N R)$ \\
\hline & $\mathrm{AH}$ & $0(N R)$ & $0(\mathrm{NR})$ & $0(N R)$ \\
\hline & $A m+A H$ & $0(\mathrm{~N} R)$ & $0(N R)$ & $0(N R)$ \\
\hline & $\mathrm{E}+\mathrm{AH}$ & $0(\mathrm{NR})$ & $0(\mathrm{NR})$ & $0(N R)$ \\
\hline
\end{tabular}

(1) NR = não repelente; RFa = repelência fraca, RFo = repelência forte; RS = repelência severa. 
que, aos 160 dias, o efeito do ácido esteárico foi positivo e significativo, quando analisado por via úmida. Provavel mente, em doses não tão el evadas, aos 160 dias, oáci do esteárico poderia trazer mai ores benefícios para a agregação.

\section{Efeito dos ciclos de umedecimento e secagem}

Os cicl os de umedecimento esecagem reduziram a agregação do solo (Quadros 6 e 7). Chaney \& Swift (1986b) chegaram a resultados que mostravam que

Quadro 6. Diâmetro médio ponderado (DMP) e diâmetro médio geométrico (DMG) dos agregados dos horizontes A e B submetidos a ciclos de umedecimento e secagem semanais, separados por via seca

\begin{tabular}{|c|c|c|c|c|c|c|c|}
\hline \multirow{2}{*}{ Horizonte } & \multirow{2}{*}{$\begin{array}{l}\text { Período de } \\
\text { incubação }\end{array}$} & \multicolumn{6}{|c|}{ Composto orgânico } \\
\hline & & $\mathbf{T}$ & $A m^{(1)}$ & $\mathbf{E}$ & $\mathbf{A H}$ & $\mathbf{A m}+\mathbf{A H}$ & $\mathbf{E}+\mathbf{A H}$ \\
\hline & dias & \multicolumn{6}{|c|}{$\mathrm{DMP}, \mathrm{mm}$} \\
\hline$A$ & $\begin{array}{r}40 \\
80 \\
160\end{array}$ & $\begin{array}{l}1,03 \\
1,06 \\
1,01\end{array}$ & $\begin{array}{l}1,82 \\
1,72 \\
1,23\end{array}$ & $\begin{array}{l}1,07 \\
1,03 \\
1,05\end{array}$ & $\begin{array}{l}2,31 \\
2,46 \\
1,90\end{array}$ & $\begin{array}{l}5,07 \\
2,64 \\
2,16\end{array}$ & $\begin{array}{l}2,21 \\
1,88 \\
1,37\end{array}$ \\
\hline \multirow[t]{2}{*}{$\mathrm{B}$} & $\begin{array}{r}40 \\
80 \\
160\end{array}$ & $\begin{array}{l}1,22 \\
1,26 \\
1,07\end{array}$ & $\begin{array}{l}1,84 \\
1,20 \\
1,13\end{array}$ & $\begin{array}{l}1,18 \\
1,27 \\
1,04\end{array}$ & $\begin{array}{r}11,29 \\
11,24 \\
6,63\end{array}$ & $\begin{array}{l}4,78 \\
6,97 \\
7,39\end{array}$ & $\begin{array}{l}6,84 \\
5,86 \\
8,19\end{array}$ \\
\hline & & \multicolumn{6}{|c|}{$\mathrm{DMG}, \mathrm{mm}$} \\
\hline A & $\begin{array}{r}40 \\
80 \\
160\end{array}$ & $\begin{array}{l}1,01 \\
1,02 \\
1,00\end{array}$ & $\begin{array}{l}1,17 \\
1,27 \\
1,05\end{array}$ & $\begin{array}{l}1,02 \\
1,01 \\
1,01\end{array}$ & $\begin{array}{l}1,29 \\
1,34 \\
1,22\end{array}$ & $\begin{array}{l}2,09 \\
1,39 \\
1,26\end{array}$ & $\begin{array}{l}1,30 \\
1,24 \\
1,09\end{array}$ \\
\hline B & $\begin{array}{r}40 \\
80 \\
160\end{array}$ & $\begin{array}{l}1,05 \\
1,05 \\
1,02\end{array}$ & $\begin{array}{l}1,16 \\
1,04 \\
1,03\end{array}$ & $\begin{array}{l}1,05 \\
1,06 \\
1,01\end{array}$ & $\begin{array}{l}4,98 \\
4,81 \\
2,50\end{array}$ & $\begin{array}{l}1,86 \\
2,66 \\
2,78\end{array}$ & $\begin{array}{l}2,58 \\
2,25 \\
3,24\end{array}$ \\
\hline
\end{tabular}

(1) $\mathrm{Am}$ = amido; $\mathrm{E}$ = ácido esteárico; $\mathrm{AH}$ = ácido húmico.

Quadro 7. Diâmetros médios ponderado e geométrico eíndice de estabilidade de agregados dos horizontes A e B submetidos a ciclos de umedecimento e secagem semanais, separados por via úmida

\begin{tabular}{|c|c|c|c|c|c|c|c|}
\hline \multirow{2}{*}{ Horizonte } & \multirow{2}{*}{$\begin{array}{l}\text { Período de } \\
\text { incubação }\end{array}$} & \multicolumn{6}{|c|}{ Composto orgânico } \\
\hline & & $\mathbf{T}^{(1)}$ & Am & $\mathbf{E}$ & AH & $\mathbf{A m}+\mathbf{A H}$ & $\mathbf{E}+\mathbf{A H}$ \\
\hline & dias & \multicolumn{6}{|c|}{ Diâmetro médio ponderado, mm } \\
\hline A & $\begin{array}{r}40 \\
80 \\
160\end{array}$ & $\begin{array}{l}0,59 \\
0,72 \\
0,69\end{array}$ & $\begin{array}{l}0,71 \\
0,79 \\
0,72\end{array}$ & $\begin{array}{l}0,74 \\
0,75 \\
0,81\end{array}$ & $\begin{array}{l}0,74 \\
0,80 \\
0,78\end{array}$ & $\begin{array}{l}0,81 \\
0,83 \\
0,83\end{array}$ & $\begin{array}{l}0,89 \\
0,90 \\
0,71\end{array}$ \\
\hline B & $\begin{array}{r}40 \\
80 \\
160\end{array}$ & $\begin{array}{l}0,48 \\
0,52 \\
0,48\end{array}$ & $\begin{array}{l}0,52 \\
0,63 \\
0,57\end{array}$ & $\begin{array}{l}0,50 \\
0,56 \\
0,58\end{array}$ & $\begin{array}{l}0,64 \\
0,63 \\
0,64\end{array}$ & $\begin{array}{l}0,67 \\
0,71 \\
0,78\end{array}$ & $\begin{array}{l}0,67 \\
0,67 \\
0,67\end{array}$ \\
\hline \multicolumn{8}{|c|}{ Diâmetro médio geométrico, mm } \\
\hline A & $\begin{array}{r}40 \\
80 \\
160\end{array}$ & $\begin{array}{l}0,46 \\
0,56 \\
0,55\end{array}$ & $\begin{array}{l}0,53 \\
0,59 \\
0,55\end{array}$ & $\begin{array}{l}0,58 \\
0,59 \\
0,62\end{array}$ & $\begin{array}{l}0,56 \\
0,60 \\
0,59\end{array}$ & $\begin{array}{l}0,59 \\
0,63 \\
0,63\end{array}$ & $\begin{array}{l}0,66 \\
0,67 \\
0,55\end{array}$ \\
\hline B & $\begin{array}{r}40 \\
80 \\
160\end{array}$ & $\begin{array}{l}0,38 \\
0,41 \\
0,40\end{array}$ & $\begin{array}{l}0,41 \\
0,50 \\
0,45\end{array}$ & $\begin{array}{l}0,40 \\
0,45 \\
0,47\end{array}$ & $\begin{array}{l}0,47 \\
0,48 \\
0,48\end{array}$ & $\begin{array}{l}0,51 \\
0,53 \\
0,59\end{array}$ & $\begin{array}{l}0,49 \\
0,49 \\
0,50\end{array}$ \\
\hline \multicolumn{8}{|c|}{ Índice de estabilidade de agregados,\% } \\
\hline A & $\begin{array}{r}40 \\
80 \\
160\end{array}$ & $\begin{array}{l}80,43 \\
92,23 \\
93,06\end{array}$ & $\begin{array}{l}84,18 \\
88,32 \\
89,92\end{array}$ & $\begin{array}{l}90,04 \\
90,02 \\
91,81\end{array}$ & $\begin{array}{l}86,00 \\
88,99 \\
91,04\end{array}$ & $\begin{array}{l}90,59 \\
93,87 \\
93,31\end{array}$ & $\begin{array}{l}92,47 \\
93,51 \\
89,13\end{array}$ \\
\hline B & $\begin{array}{r}40 \\
80 \\
160\end{array}$ & $\begin{array}{l}72,15 \\
73,68 \\
76,23\end{array}$ & $\begin{array}{l}76,26 \\
87,44 \\
80,79\end{array}$ & $\begin{array}{l}72,07 \\
83,81 \\
86,38\end{array}$ & $\begin{array}{l}80,47 \\
89,09 \\
82,47\end{array}$ & $\begin{array}{l}84,91 \\
83,21 \\
90,62\end{array}$ & $\begin{array}{l}79,70 \\
80,46 \\
84,31\end{array}$ \\
\hline
\end{tabular}

(1) $\mathrm{T}$ = testemunha; $\mathrm{Am}=$ amido; $\mathrm{E}$ =ácido esteárico; $\mathrm{AH}$ =ácido húmico. 
os ciclos de umedecimento e secagem não mel horaram a estabilidade de agregados anal isados por via úmida.

O efeito dos ciclos de umedecimento e secagem sobre os tratamentos com compostos orgânicos, praticamente, só influenciaram, de forma significativa e rel evante, o horizonte B (Quadro 8). Acredita-se que isso se dê pelo fato de os compostos orgânicos utilizados sofrerem ação intensa desses, diminuindo seu efeito agregante. Esseresultado foi mais evidenciado no horizonte $A$, no qual já havia uma agregação inicial maior do que no horizonte $B$.

Nohorizonte B, tanto o contraste do amido, ácido húmico e a combinação destes versus a testemunha, quanto o contraste do áci do esteárico, ácido húmico e combinação dos dois versus testemunha tiveram comportamentos similares (Quadro 8). A estabilidade dos agregados cresce com o tempo, quando o solo é analisado por via úmida, e cai com o tempo, quando analisado por via seca. Isto reforça a idéia de que o

Quadro 8. Contrastes médios para as variáveis analisadas no experimento de ciclos de umedecimento e secagem

\begin{tabular}{|c|c|c|c|c|c|}
\hline Contraste & DMPS & DMGS & DMPU & DMGU & IEAU \\
\hline & \multicolumn{4}{|c|}{$-\mathrm{mm}$} & $\%$ \\
\hline$\overline{\mathrm{C}} 1 \mathrm{~d} / \mathrm{T}_{40} \mathrm{~d} / \mathrm{A}$ & $2,04 * *$ & 0,51 & 0,17 & $0,10 *$ & $6,49^{\circ}$ \\
\hline$\overline{\mathrm{C}} 1 \mathrm{~d} / \mathrm{T}_{80} \mathrm{~d} / \mathrm{A}$ & $1,21^{\circ}$ & 0,31 & 0,09 & 0,05 & $-1,83$ \\
\hline $\mathrm{C} 1 \mathrm{~d} / \mathrm{T}_{160} \mathrm{~d} / \mathrm{A}$ & 0,75 & 0,18 & 0,09 & 0,04 & 1,64 \\
\hline$\overline{\mathrm{C}} 2 \mathrm{~d} / \mathrm{T}_{40} \mathrm{~d} / \mathrm{A}$ & $3,00 * *$ & $0,86 * *$ & $0,09 * *$ & 0,05 & 5,50 \\
\hline$\overline{\mathrm{C}} 2 \mathrm{~d} / \mathrm{T}_{80} \mathrm{~d} / \mathrm{A}$ & 0,55 & 0,09 & 0,04 & 0,04 & 5,22 \\
\hline $\bar{C} 2 \mathrm{~d} / \mathrm{T}_{160} \mathrm{~d} / \mathrm{A}$ & 0,60 & 0,13 & $0,08^{*}$ & $0,06^{\circ}$ & 2,83 \\
\hline$\overline{\mathrm{C}} 3 \mathrm{~d} / \mathrm{T}_{40} \mathrm{~d} / \mathrm{A}$ & 0,49 & 0,12 & 0,03 & 0,03 & 1,82 \\
\hline C $3 \mathrm{~d} / \mathrm{T}_{80} \mathrm{~d} / \mathrm{A}$ & 0,74 & 0,07 & 0,01 & 0,01 & 0,67 \\
\hline $\bar{C} 3 \mathrm{~d} / \mathrm{T}_{160} \mathrm{~d} / \mathrm{A}$ & 0,67 & 0,17 & 0,06 & 0,04 & 1,12 \\
\hline $\mathrm{C} 1 \mathrm{~d} / \mathrm{T}_{40} \mathrm{~d} / \mathrm{B}$ & $4,75^{* *}$ & $1,61^{* *}$ & $0,13^{*}$ & $0,08 *$ & $8,40^{*}$ \\
\hline$\overline{\mathrm{C}} 1 \mathrm{~d} / \mathrm{T}_{80} \mathrm{~d} / \mathrm{B}$ & $5,21^{* *}$ & $1,79 * *$ & $0,14^{*}$ & $0,09 *$ & $12,90 * *$ \\
\hline$\overline{\mathrm{C}} 1 \mathrm{~d} / \mathrm{T}_{160} \mathrm{~d} / \mathrm{B}$ & $3,98 * *$ & $1,08^{* *}$ & $0,18^{* *}$ & $0,11^{* *}$ & $8,40^{*}$ \\
\hline $\bar{C} 2 \mathrm{~d} / \mathrm{T}_{40} \mathrm{~d} / \mathrm{B}$ & $-1,79^{*}$ & $-1,21 * *$ & 0,09 & $0,07^{\circ}$ & $6,55^{\circ}$ \\
\hline$\overline{\mathrm{C}} 2 \mathrm{~d} / \mathrm{T}_{80} \mathrm{~d} / \mathrm{B}$ & 0,75 & $-0,27$ & 0,08 & 0,04 & $-5,06$ \\
\hline $\bar{C} 2 \mathrm{~d} / \mathrm{T}_{160} \mathrm{~d} / \mathrm{B}$ & $3,51^{* *}$ & $1,02 * *$ & $0,18^{*}$ & $0,13^{* *}$ & $8,99 *$ \\
\hline$\overline{\mathrm{C}} 3 \mathrm{~d} / \mathrm{T}_{40} \mathrm{~d} / \mathrm{B}$ & $9,45^{* *}$ & $3,82 * *$ & 0,12 & $0,06^{\circ}$ & 4,21 \\
\hline $\mathrm{C} 3 \mathrm{~d} / \mathrm{T}_{80} \mathrm{~d} / \mathrm{B}$ & $10,04 * *$ & $3,77^{* *} *$ & 0,00 & $-0,02$ & 1,65 \\
\hline $\bar{C} 3 \mathrm{~d} / \mathrm{T}_{160} \mathrm{~d} / \mathrm{B}$ & $5,50 * *$ & $1,47^{* *}$ & 0,07 & 0,03 & 1,68 \\
\hline $\mathrm{C} 4 \mathrm{~d} / \mathrm{T}_{40} \mathrm{~d} / \mathrm{A}$ & 0,83 & 0,19 & 0,02 & $0,14^{* *}$ & $9,07 *$ \\
\hline $\bar{C} 4 \mathrm{~d} / \mathrm{T}_{80} \mathrm{~d} / \mathrm{A}$ & 0,73 & 0,18 & 0,10 & $0,06^{\circ}$ & $-1,39$ \\
\hline $\bar{C} 4 \mathrm{~d} / \mathrm{T}_{160} \mathrm{~d} / \mathrm{A}$ & 0,43 & 0,11 & 0,08 & 0,04 & $-2,40$ \\
\hline $\bar{C} 5 \mathrm{~d} / \mathrm{T}_{40} \mathrm{~d} / \mathrm{A}$ & 0,52 & 0,15 & $0,15^{*}$ & $0,09 *$ & 4,45 \\
\hline $\bar{C} 5 \mathrm{~d} / \mathrm{T}_{80} \mathrm{~d} / \mathrm{A}$ & 0,14 & 0,07 & $0,13^{\circ}$ & $0,08^{\circ}$ & 4,00 \\
\hline C $5 \mathrm{~d} / \mathrm{T}_{160} \mathrm{~d} / \mathrm{A}$ & $-0,11$ & $-0,03$ & 0,09 & $-0,06^{\circ}$ & $-2,48$ \\
\hline $\bar{C} 6 \mathrm{~d} / \mathrm{T}_{40} \mathrm{~d} / \mathrm{A}$ & 1,24 & 0,27 & 0,00 & $-0,02$ & $-4,04$ \\
\hline $\bar{C} 6 \mathrm{~d} / \mathrm{T}_{80} \mathrm{~d} / \mathrm{A}$ & $1,43^{\circ}$ & 0,33 & 0,05 & 0,01 & $-1,03$ \\
\hline $\bar{C} 6 \mathrm{~d} / \mathrm{T}_{160} \mathrm{~d} / \mathrm{A}$ & 0,85 & 0,21 & $-0,03$ & $-0,03$ & $-0,77$ \\
\hline$\overline{\mathrm{C}} 4 \mathrm{~d} / \mathrm{T}_{40} \mathrm{~d} / \mathrm{B}$ & $5,22 * *$ & $1,82 * *$ & $0,12^{\circ}$ & $0,07^{\circ}$ & 5,26 \\
\hline$\overline{\mathrm{C}} 4 \mathrm{~d} / \mathrm{T} 80 \mathrm{~d} / \mathrm{B}$ & $4,86 * *$ & $1,66 * *$ & 0,10 & $0,06^{\circ}$ & $10,77 * *$ \\
\hline$\overline{\mathrm{C}} 4 \mathrm{~d} / \mathrm{T}_{160} \mathrm{~d} / \mathrm{B}$ & $4,22 * *$ & $1,23^{* *}$ & $0,15^{*}$ & $0,08 *$ & $8,16^{*}$ \\
\hline$\overline{\mathrm{C}} 5 \mathrm{~d} / \mathrm{T}_{40} \mathrm{~d} / \mathrm{B}$ & 0,61 & $-0,44$ & 0,10 & $0,05^{\circ}$ & 3,43 \\
\hline$\overline{\mathrm{C}} 5 \mathrm{~d} / \mathrm{T}_{80} \mathrm{~d} / \mathrm{B}$ & $-0,40$ & $-0,69 *$ & 0,08 & 0,03 & $-5,99$ \\
\hline$\overline{\mathrm{C}} 5 \mathrm{~d} / \mathrm{T}_{160} \mathrm{~d} / \mathrm{B}$ & $4,36 * *$ & $1,49 * *$ & 0,06 & 0,03 & $-0,12$ \\
\hline $\mathrm{C} 6 \mathrm{~d} / \mathrm{T}_{40} \mathrm{~d} / \mathrm{B}$ & $10,11 * *$ & $3,93 * *$ & $0,14^{\circ}$ & $0,07^{\circ}$ & $8,40^{\circ}$ \\
\hline $\mathrm{C} 6 \mathrm{~d} / \mathrm{T}_{80} \mathrm{~d} / \mathrm{B}$ & $9,97 * *$ & $3,75^{* *}$ & 0,07 & 0,03 & 5,28 \\
\hline$\overline{\mathrm{C}} 6 \mathrm{~d} / \mathrm{T} 160 \mathrm{~d} / \mathrm{B}$ & $5,59 * *$ & $1,49 * *$ & 0,06 & 0,01 & $-3,91$ \\
\hline Resíduo $(72)^{+}$ & $1,00^{++}$ & $0,19++$ & $0,01^{++}$ & $0,003^{++}$ & $28,16^{++}$ \\
\hline C.V. (\%) & 31,71 & 26,74 & 10,86 & 10,71 & 6,20 \\
\hline
\end{tabular}

DMPS = diâmetro médio ponderado via seca; DMGS = diâmetro médio geométrico via seca; DMPU = diâmetro médio ponderado via úmida; DMGU = diâmetro médio geométrico via úmida; IEAU = índice de estabilidade de agregados via úmida; $\overline{\mathrm{C}} 1=[-$ $3 \mathrm{~T}+\mathrm{Am}+\mathrm{AH}+(\mathrm{Am}+\mathrm{AH})] / 3 ; \mathrm{C} 2=[-\mathrm{Am}-\mathrm{AH}+2(\mathrm{Am}+\mathrm{AH})] / 2 ; \mathrm{C} 3=(-\mathrm{Am}+\mathrm{AH}) / 1 ; \mathrm{C} 4=[-3 \mathrm{~T}+\mathrm{E}+\mathrm{AH}+(\mathrm{E}+\mathrm{AH})] / 3 ; \mathrm{C} 5=[-\mathrm{E}-$ $\mathrm{AH}+2(\mathrm{E}+\mathrm{AH})] / 2 ; \overline{\mathrm{C}} 6=(-\mathrm{E}+\mathrm{AH}) / 1 ; \mathrm{d} /$ - dentro de; $\mathrm{T}_{40}, \mathrm{~T}_{80}$ e $\mathrm{T}_{160}=$ período de incubação de 40,80 e 160 dias, respectivamente; $\mathrm{A}$ $=$ horizonte $\mathrm{A} ; \mathrm{B}=$ horizonte $\mathrm{B} ;{ }^{\circ}, * \mathrm{e}^{* *}=$ contraste significativo a 10,5 e $1 \%$ pelo teste $\mathrm{F}$, respectivamente; $+\mathrm{e}++=$ grau de liberdade e quadrado médio, respectivamente. 
composto hidrofílico (amido) ou hidrofóbico (ácido esteárico) não tiveram efeito marcante na agregação, quando expostos a cicl os de umedecimento e secagem semanais.

Os contrastes que comparam o amido ou o ácido esteárico com o áci do húmico mostram que o maior responsável pela estabilização dos agregados foi o ácido húmico. Contudo, seu efeito foi diminuído quando exposto a um maior número de ciclos de umedecimento e secagem.

O grau de repelência à água não mostrou nenhuma relação com as respostas dos efeitos estudados tanto na análise via seca quanto na via úmida (Quadros 5 e 8). Nenhum tratamento apresentou repelência à água. Pode-se inferir que os ciclos de umedecimento e secagem inibiram a repelência à água existente no solo tratado com compostos orgânicos hidrofóbicos, aumentando o efeito da água na ruptura dos agregados.

\section{CONCLUSÕES}

1. Os compostos orgânicos com caráter hidrofóbico acentuado tiveram efeito positivo e significativo na melhoria da estabilidade dos agregados em água, tendo sua mel hor resposta na dose de $5,5 \mathrm{~g} \mathrm{~kg}^{-1}$.

2. Os ciclos de umedecimento e secagem reduziram os efeitos dos compostos orgânicos adicionados sobre a agregação do solo.

\section{LITE RATURA CITADA}

ANGERS, D.A. \& MEHUYS, G.R. Effects of cropping on carbohydrate content and water stable aggregation of a clay soil. Can. J. Soil Sci., 69:373-380, 1989.

BASTOS, R.S.; SÁ MENDONÇA, E.; ALVAREZ V., V.H. \& CORRÊA, M.M. Formação e estabilização de agregados do solo decorrentes da adição de compostos orgânicos com diferentes características hidrofóbicas. R. Bras. Ci. Solo, 29:11-20, 2005.

BAVER, L.D. The effect of organic matter on soil structure. In: SEMAINE D'ETUDE SUR LE THEME MATIERE ORGANIQUE ET FERTILITE DU SOL., Amsterdam, 1968. Semaine... Vaticano, Pontificia Academia Scientiarum, 1968. p.383-404.

CARVALHO, A.F. Emprego da agitação horizontal na avaliação da estabilidade de agregados de cinco solos da região sudeste. Viçosa, U niversidade Federal de Viçosa, 1991. 73p. (Tese de Mestrado)

CASTRO FILHO, C.; MUZILLI, O. \& PODANOSCHI, A.L. Estabilidade dos agregados e sua relação com o teor de carbono orgânico num Latossolo Roxo distrófico, em função de sistemas de plantio, rotações de culturas e métodos de preparo de amostras. R. Bras. Ci. Solo, 22:527-538, 1998.
CHANEY, K. \& SWIFT, R.S. The influence of organic matter on the stability of some British soils. J. Soil Sci., 35:223230, 1984.

CHANEY, K. \& SWIFT, R.S. Studies on aggregate stability. I. Re-formation of soil aggregates. J. Soil Sci., 37:329-335, 1986a.

CHANEY, K. \& SWIFT, R.S. Studies on aggregate stability. II. The effect of humic substances on the stability of re-formed soil aggregates. J . Soil Sci., 37:337-343, 1986b.

DEXTER, A.R. Advances in characterization of soil structure. Soil \& Till. Res., 11:199-238, 1988.

DINEL, H.; LEVESQUE, M. \& MEHUYS, G.R. Effects of long chain aliphatic compounds on the aggregate stability of a lacustrine silty clay. Soil Sci., 151:228-239, 1991a.

EDWARDS, A.P. \& BREMNER, J.M. Microaggregates in soils. J. Soil Sci., 18:64-73, 1967.

EMERSON, W.W. The structure of soil crumbs. J . Soil Sci., 10:235-244, 1959.

HAYNES, R.J . \& SWIFT, R.S. Stability of soil aggregates in relation to organic constituents and soil water content. J . Soil Sci., 41:73-83, 1990.

HAYES, M.H. \& MALCOLM, R.L. Considerations of compositions and of aspects of the structures of humic substances. In: HAYES, M.H. \& MALCOLM, R.L., eds. Humic Substances and Chemical Contaminants. Madison, Soil Science Society of America, 2001. 750p.

HILLEL, D. Fundamentals of soil physics. New York, Academic Press, 1980. 413p.

KEMPER, W.D. \& CHEPIL, W.S. Sizedistribution of aggregates. In: BLACK, C.A., ed. Methods of soil analysis. Madison, American Society of Agronomy, 1965. p.449-510.

KING, P.M. Comparison of methods for measuring severity of water repellence of sandy soils and assessment of some factors that affect its measurement. Aust. J . Soil Res., 19:275-285, 1981.

LETEY, J. Measurement of contact angle, water drop penetration time and critical surface tension. In: SYMPOSIUM WATER REPORT SOILS, Riverside, 1968. Proceedings. University of California, 1969. p.43-48.

MALTONI, K.L. Estudo da compactação e, ou, adensamento em subsuperfície de latossol os sob diferentes usos. Viçosa, Universidade Federal de Viçosa, 1994. 73p. (Tese de Doutorado)

OADES, J .M. Soil organic matter and structural stability: mechanisms and implications for management. Plant Soil, 76:319-337, 1984.

OADES, J.M. \& WATERS, S.G. Aggregate hierarchy in soils. Aust. J . Soil Res., 29:815-828, 1991.

PICCOLO, A. \& MBAGWU, J.S.C. Role of hydrophobic components of soil organic matter in soil aggregatestability. Soil Sci. Soc. Am. J ., 63:1081-1810, 1999.

SILVA, I.F. \& MIELNICZUK, J. Ação do sistema radicular de plantas na formação e estabilização de agregados do solo. R. Bras. Ci. Solo, 22:113-117, 1998. 
SIX, J .; PAUSTIAN, K.; ELLIOTT, E.T. \& COMBRINK, C. Soil structure and organic matter: I. Distribution of aggregatesize classes and aggregate-associated carbon. Soil Sci. Soc. Am. J ., 64:681-689, 2000.

STEVENSON, F.J. \& COLE, M.A. Cycles of soil: carbon, nitrogen, phosporus, sulfur and micronutrients. New York, J ohn Wiley \& Sons, 1999. 427p.
TISDALL, J .M. \& OADES, J .M. Organic matter and waterstable aggregates in soil. J . Soil Sci., 33:141-163, 1982.

UNIVERSIDADE FEDERAL DE VIÇOSA - UFV. SAEG Sistema para análise estatística v.5.0. Viçosa, 1993.

YODER, R.E. A direct method of aggregate analysis of soil and a study of the physical nature erosion losses. J . Am. Soc. Agric., 28:337-351, 1936. 